\title{
ON CONTINUED FRACTIONS OF GIVEN PERIOD
}

\author{
CHRISTIAN FRIESEN
}

(Communicated by Larry J. Goldstein)

\begin{abstract}
A direct proof is given of the fact that, for any $k \in \mathbf{Z}^{+}$, there are infinitely many squarefree integers $N$, where the continued fraction expansion of $\sqrt{N}$ has period equal to $k$.
\end{abstract}

I. Introduction. In Problems on periodic simple continued fractions [1], Chowla and Chowla exhibit several conjectures concerning continued fraction expansions. In particular, one conjecture is the strong statement that, for any positive integer $k$, there exist infinitely many prime $N$ with the continued fraction expansion of $\sqrt{N}$ having period $k$. Resolving this conjecture in the affirmative would show that, for example, in the case $k=1$, there are infinitely many primes of the form $n^{2}+1, n \in \mathbf{Z}$, which is a longstanding unsolved problem.

It is, however, not difficult to show that, for any positive integer $k$, there exist infinitely many squarefree $N$ with a continued fraction expansion of period $k$. This fact is a simple corollary of a more general result to be proved in this paper.

Let the continued fraction expansion of $\sqrt{N}, N$ a nonsquare integer, be written $\sqrt{N}=\left(a_{0} ; \overline{a_{1}, a_{2}, \ldots, a_{k-1}, a_{k}=2 a_{0}}\right)$, where $k$ is the period and the $a_{i}(0 \leq i \leq k)$ are the partial denominators. This is the same as the following:

$$
\begin{aligned}
\sqrt{N}=a_{0} \frac{1}{a_{1} \frac{1}{a_{2}+1} \frac{1}{a_{3}+\cdots}} & \\
\cdots & \frac{+1}{a_{k-2}+1} \frac{a_{k-1} \frac{1}{\sqrt{N}+a_{0}}}{}
\end{aligned}
$$

Fix $k \in \mathbf{Z}^{+}$. Given a set of positive integers $\left\{a_{n}\right\}_{n=0,1, \ldots, k-1}$ we define

$$
\begin{array}{ll}
P_{-1}=1, & Q_{-1}=0, \\
P_{0}=a_{0}, & Q_{0}=1, \\
P_{n}=a_{n} P_{n-1}+P_{n-2}, & Q_{n}=a_{n} Q_{n-1}+Q_{n-2} \quad \text { for } n=1,2, \ldots, k-1 .
\end{array}
$$

Received by the editors July 15, 1986 and, in revised form, September 23, 1986.

1980 Mathematics Subject Classification (1985 Revision). Primary 11A55.

The author wishes to acknowledge the support of a Natural Sciences and Research Council of Canada 1967 Scholarship held while writing this paper. 


\section{Results.}

THEOREM. Let $[N]$ denote the greatest integer $\leq \sqrt{N}$. Then the equation

$$
\sqrt{N}=\left([\sqrt{N}] ; \overline{a_{1}, a_{2}, \ldots, a_{k-2}=a_{2}, a_{k-1}=a_{1}, a_{k}=2[\sqrt{N}]}\right)
$$

has, for any symmetric set of positive integers $\left\{a_{1}, \ldots, a_{k-1}\right\}$, infinitely many squarefree solutions $N$ whenever either $Q_{k-2}$ or $\left(Q_{k-2}^{2}-(-1)^{k}\right) / Q_{k-1}$ is even. If both quantities are odd, then there are no solutions $N$ even if the squarefree condition is dropped.

COROLLARY. For any positive integer $k$ there exist infinitely many squarefree integers $N$ with a continued fraction expansion of period $k$.

Proof of the Theorem and Corollary will follow as a result of three lemmas that are set out below. To simplify the presentation it will be assumed that $k>1$. The special case $k=1$ will be treated separately.

LEMMA 1. With $P_{n}$ and $Q_{n}$ defined as before we have

$$
\begin{aligned}
& a_{0}+1 \\
& \overline{a_{1}+1} \frac{1}{a_{2}+\cdots} \\
& \ldots \quad=\frac{x P_{k-1}+P_{k-2}}{x Q_{k-1}+Q_{k-2}} \quad \text { where } x \in \mathbf{R}^{+} \text {, } \\
& \cdots \frac{+1}{a_{k-1} \frac{+1}{x}}
\end{aligned}
$$

as well as the identity

$$
P_{n} Q_{n-1}-P_{n-1} Q_{n}=(-1)^{n+1} \text { for } n=0,1, \ldots k-1 .
$$

PROOF. The proof of Lemma 1 is by induction and appears in standard texts on continued fractions. See, for example, [2].

Consider the following formula, which we arrive at by setting $x=\sqrt{N}+a_{0}$ in the equation in Lemma 1 :

$$
\begin{aligned}
& a_{0} \frac{1}{a_{1} \frac{1}{a_{2}+\cdots}} \\
& \cdots \quad=\frac{\left(\sqrt{N}+a_{0}\right) P_{k-1}+P_{k-2}}{\left(\sqrt{N}+a_{0}\right) Q_{k-1}+Q_{k-2}} \\
& \cdots \frac{+1}{a_{k-1} \frac{+1}{\sqrt{N}+a_{0}}}
\end{aligned}
$$

For the left-hand side of the above formula to equal $\sqrt{N}$ (ensuring that $\sqrt{N}=$ $\left.\left(a_{0} ; \overline{a_{1}, a_{2}, \ldots, a_{k-1}, a_{k}=2 a_{0}}\right)\right)$ with $N$ a nonsquare integer, we equate both the 
integer parts and coefficients of $\sqrt{N}$ in the equation obtained by setting the righthand side equal to $\sqrt{N}$. This gives

$$
P_{k-1}=a_{0} Q_{k-1}+Q_{k-2} \text { and } N Q_{k-1}=a_{0} P_{k-1}+P_{k-2} .
$$

From the identity in Lemma 1 , with $n=k-1$, we get

$$
P_{k-2}=\left(P_{k-1} Q_{k-2}-(-1)^{k}\right) / Q_{k-1} \text {. }
$$

These three equations give us, as a sufficient condition for $N$ to be an integer with $\sqrt{N}$ having a continued fraction expansion with period $=k$, the following:

$$
\left(N-a_{0}^{2}\right) Q_{k-1}-\left(2 a_{0}\right) Q_{k-2}=\left(Q_{k-2}^{2}-(-1)^{k}\right) / Q_{k-1} .
$$

A simple induction with the defining formula for the $Q_{n}$ 's shows that $Q_{k-1}$ and $Q_{k-2}$ are coprime. Hence the integer solutions $x$ and $y$ of

$$
x Q_{k-1}-y Q_{k-2}=z, \quad \text { with } z \text { any integer },
$$

are exactly those of the form

$$
\begin{aligned}
& x=(-1)^{k+1} z\left(Q_{k-2}^{2}-(-1)^{k}\right) / Q_{k-1}+m Q_{k-2} \text { and } \\
& y=(-1)^{k+1} z Q_{k-2}+m Q_{k-1}, \quad \text { where } m \text { is any integer. }
\end{aligned}
$$

So, $(*)$ is equivalent to the following:

$$
\begin{aligned}
& N-a_{0}^{2}=(-1)^{k+1}\left(Q_{k-2}^{2}-(-1)^{k}\right)^{2} / Q_{k-1}^{2}+m Q_{k-2} \text { and } \\
& 2 a_{0}=(-1)^{k+1} Q_{k-2}\left(Q_{k-2}^{2}-(-1)^{k}\right) / Q_{k-1}+m Q_{k-1} .
\end{aligned}
$$

We must, in order to have $a_{0}$ positive, require that $m \geq m_{0}$, where $m_{0}$ is the smallest integer for which

$$
(-1)^{k+1} Q_{k-2}\left(Q_{k-2}^{2}-(-1)^{k}\right)+m_{0} Q_{k-1}^{2}>0 .
$$

This condition on $m$ also suffices to ensure that $N-a_{0}^{2}>0$ and that $2 a_{0} \geq N-a_{0}^{2}$, allowing $a_{0}$ to equal $[\sqrt{N}]$. The only further condition that needs to be met is the requirement that the expression for $2 a_{0}$ has, in fact, even parity so that $a_{0} \in \mathbf{Z}$. We shall divide the possibilities into four cases.

Case $1 . Q_{k-2} \equiv 0(\bmod 2)$. In this case $\left(Q_{k-2}, Q_{k-1}\right)=1 \Rightarrow Q_{k-1} \equiv 1(\bmod 2)$ and the even $m$ are the only solutions that will force $2 a_{0} \equiv 0(\bmod 2)$.

Case 2. $Q_{k-2} \equiv Q_{k-1} \equiv 1(\bmod 2)$. In this case the even $m$ are the only solutions.

Case 3. $Q_{k-2} \equiv 1(\bmod 2), Q_{k-1} \equiv\left(Q_{k-2}^{2}-(-1)^{k}\right) / Q_{k-1} \equiv 0(\bmod 2)$. Here all $m$ satisfy the congruence $2 a_{0} \equiv 0(\bmod 2)$.

Case 4. $Q_{k-2} \equiv\left(Q_{k-2}^{2}-(-1)^{k}\right) / Q_{k-1} \equiv 1(\bmod 2)$. This implies that $Q_{k-1} \equiv$ $0(\bmod 2)$ and there are no solutions $m \in \mathbf{Z}$ which make the formula for $2 a_{0}$ come out even.

Case 4, showing the impossibility of any solutions when both $Q_{k-2}$ and $\left(Q_{k-2}^{2}-(-1)^{k}\right) / Q_{k-1}$ are odd, proves the second statement of the theorem. For Cases 1 and 2 we see that $N$ has a continued fraction expansion of the desired form 
exactly when

$$
\begin{aligned}
N= & \left\{(-1)^{k+1} Q_{k-2}\left(Q_{k-2}^{2}-(-1)^{k}\right) / 2 Q_{k-1}+b Q_{k-1}\right\}^{2}+2 b Q_{k-2} \\
& +(-1)^{k+1}\left(Q_{k-2}^{2}-(-1)^{k}\right)^{2} / Q_{k-1}^{2} \text { for } b \in \mathbf{Z} \text { and } b \geq b_{0}=m_{0} / 2 \\
= & \alpha b^{2}+\beta b+\gamma \text { with integral coefficients, } \\
\alpha= & Q_{k-1}^{2}, \\
\beta= & 2 Q_{k-2}-(-1)^{k} Q_{k-2}\left(Q_{k-2}^{2}-(-1)^{k}\right), \\
\gamma= & \left(Q_{k-2}^{2} / 4-(-1)^{k}\right)\left(Q_{k-2}^{2}-(-1)^{k}\right)^{2} / Q_{k-1}^{2} .
\end{aligned}
$$

Note that the discriminant $\delta=\beta^{2}-4 \alpha \gamma=4(-1)^{k}$.

For case 3 we see that $N$ has a continued fraction expansion of the desired form exactly when

$$
N=\alpha b^{2}+\beta b+\gamma \quad \text { for } b \in \mathbf{Z} \text { and } b \geq b_{0}=m_{0},
$$

where the integral coefficients are

$$
\begin{aligned}
& \alpha=Q_{k-1}^{2} / 4, \\
& \beta=Q_{k-2}-(-1)^{k} Q_{k-2}\left(Q_{k-2}^{2}-(-1)^{k}\right) / 2, \\
& \gamma=\left(Q_{k-2}^{2} / 4-(-1)^{k}\right)\left(Q_{k-2}^{2}-(-1)^{k}\right)^{2} / Q_{k-1}^{2} .
\end{aligned}
$$

Here we have $\delta=\beta^{2}-4 \alpha \gamma=(-1)^{k}$. In fact, looking at $\delta$ modulo 4 shows that $\delta$ must equal 1 and that $k$ must be even for this case to arise.

In summary then we have that all $N$, and only those, of the form

$$
N=N(b)=\alpha b^{2}+\beta b^{2}+\gamma, \quad b \in \mathbf{Z}, b \geq b_{0},
$$

are solutions to $\sqrt{N}=\left([\sqrt{N}] ; \overline{a_{1}, a_{2}, \ldots, a_{k-2}=a_{2}, a_{k-1}=a_{1}, a_{k}=2[\sqrt{N}]}\right)$ for some integers $\alpha, \beta$, and $\gamma$ dependent only on $a_{1}, a_{2}, \ldots, a_{k-1}$. A notationally different and less direct proof of a similar result may be found in [3, pp. 96-99].

For the special case $k=1$, we have

$$
\sqrt{N}=a_{0}+1 /\left(\sqrt{N}+a_{0}\right) \Rightarrow N=a_{0}^{2}+1
$$

allowing us to write, as a solution set,

$$
\left\{N(b)=\alpha b^{2}+\beta b+\gamma \mid b \in \mathbf{Z}, b \geq b_{0}=1\right\},
$$

where $\alpha=1, \beta=0$, and $\gamma=1$. In this instance, $\delta=\beta^{2}-4 \alpha \gamma=-4$. We are now ready for

LEMMA 2. With notation as above we have:

For any prime $p, N(n) \equiv 0\left(\bmod p^{2}\right)$ for at most two $n$ in any residue system modulo $p$.

ProOF. First consider $p=2$. As $n$ goes through a complete residue system modulo $4, N(n)$ takes on the values $\gamma, \alpha+\beta+\gamma, 2 \beta+\gamma$, and $\alpha-\beta+\gamma$. But if $Q_{k-1} \equiv 1(\bmod 2)$ we have $\alpha \equiv 1(\bmod 2)$ and if $Q_{k-1} \equiv 0(\bmod 2)$ then $\delta=(-1)^{k}$ implies that $\beta \equiv 1(\bmod 2)$. Using this information one may inspect the four values to verify that at most two of them can be congruent to 0 modulo 4 .

Now let $p$ be an odd prime. Assume $p \mid N(b)$, say $N(b) \equiv l p\left(\bmod p^{2}\right)$. Then $N(b+n p) \equiv 2 \alpha b n p+\beta n p+l p\left(\bmod p^{2}\right)$. We have $N(b+n p) \equiv 0\left(\bmod p^{2}\right) \Leftrightarrow$ $l+(2 \alpha b+\beta) n \equiv 0(\bmod p)$. But $N(b) \equiv 0(\bmod p)$ together with $p \nmid \delta$ implies 
$p \nmid 2 \alpha b+\beta$ and so $(2 \alpha b+\beta)$ is invertible modulo $p$. So $l+(2 \alpha b+\beta) n \equiv 0(\bmod p)$ for exactly one choice of $n$ modulo $p$. However, $p \mid N(b)$ is possible for at most two choices of $b(\bmod p)$ as $p+\delta \Rightarrow \alpha x^{2}+\beta x+\gamma$ is not identically congruent to 0 $\bmod p$. Hence there exist at most two $n$ in any residue system modulo $p^{2}$ such that $N(n) \equiv 0\left(\bmod p^{2}\right)$.

We are now ready to conclude the proof of the Theorem with one last lemma which will show that the density of $b$ in $\mathrm{Z}^{+}$such that $N(b)$ is squarefree is nonzero.

LEMMA 3. Define the density $D=\lim _{n \rightarrow \infty}$ (number of squarefree $N(b)$ with $b \leq n) / n$. Then $D>0$.

PROOF.

$$
\begin{aligned}
D & \geq \prod_{p \text { prime }}\left(1-\frac{2}{p^{2}}\right) \geq \prod_{n=2}^{\infty}\left(1-\frac{2}{n^{2}}\right)=\exp \left\{\sum_{n=2}^{\infty} \ln \left(1-\frac{2}{n^{2}}\right)\right\} \\
& \geq \exp \left\{\sum_{n=2}^{\infty} \frac{-4}{n^{2}}\right\} \text { as } \ln \left(1-2 / n^{2}\right)>-4 / n^{2} \text { for } n \geq 2, \\
& \geq \exp \left\{-4 \pi^{2} / 6\right\}>0 .
\end{aligned}
$$

This completes the proof of the lemma and finishes off the proof of the main Theorem. To prove the Corollary it will suffice to exhibit, for each $k \in \mathbf{Z}^{+}$, a symmetric set of positive integers $\left\{a_{1}, \ldots, a_{k-1}\right\}$ such that $Q_{k-1}$ is odd (thus ensuring that one of $Q_{k-2}$ or $\left(Q_{k-2}^{2}-(-1)^{k}\right) / Q_{k-1}$ is even).

If $k=1$ then $N(b)=b^{2}+1$ gives us infinitely many squarefree $N$. Assume $k>1$. If $k \not \equiv 0(\bmod 3)$ then set $a_{1}=a_{k-1}=2$ and $a_{i}=1$ for $i=2, \ldots, k-2$. If $k \equiv 0(\bmod 3)$ set $a_{i}=1$ for $i=1, \ldots, k-1$. In both cases we have the recursion formula for $Q_{n}$ giving us copies of the Fibonacci sequence $\left(F_{1}=F_{2}=1\right.$ and $F_{n}=F_{n-1}+F_{n-2}$ for $n \geq 3$ define the sequence). In the first instance we have $Q_{n}=F_{n+2}$ for $i=1, \ldots, k-2$ and $Q_{k-1}=2 Q_{k-2}+Q_{k-3}=2 F_{k}+F_{k-1}+F_{k+2}$. If $k \not \equiv 0(\bmod 3)$ we have $Q_{n}=F_{n+1}$ for $n=1, \ldots, k-1$, hence $Q_{k-1}=F_{k}$. But, as $F_{k}$ is even only when $k \equiv 0(\bmod 3)$, we see that $Q_{k-1}$ is odd in either situation. Therefore, either $Q_{k-2}$ or $\left(Q_{k-2}^{2}-(-1)^{k}\right) / Q_{k-1}$ is even and we have satisfied the conditions of the Theorem, thus proving the Corollary.

III. Conclusion. We have shown that, given any symmetric set of positive integers $\left\{a_{1}, a_{2}, \ldots, a_{k-2}=a_{2}, a_{k-1}=a_{1}\right\}$ we can find either no integers $N$ (and $\left.a_{0}=[\sqrt{N}]\right)$ where $\sqrt{N}=\left(a_{0} ; \overline{a_{1}, a_{2}, \ldots, a_{k-1}, a_{k}=2 a_{0}}\right)$ or we can find infinitely many, distinguishing between the cases by inspecting $Q_{k-1}$ and $Q_{k-2}$. When there are solutions $N$ they correspond exactly to the positive values taken by a quadratic (with domain $\mathbf{Z}$ ) whose integer coefficients turn out to be functions only of $Q_{k-1}$ and $Q_{k-2}$. Among these values there must be infinitely many squarefree integers.

By setting $a_{i}=1$ for $i=1, \ldots, k-1$ if $k \not \equiv 0(\bmod 3)$ [and $a_{1}=a_{k-1}=2$, $a_{i}=1$ for $i=2, \ldots, k-2$ if $\left.k \equiv 0(\bmod 3)\right]$ it was shown that the conditions of the Theorem are met. Hence, for any $k \in \mathbf{Z}^{+}$, there are infinitely many squarefree integers $N$ with period equal to $k$. 


\section{REFERENCES}

1. P. Chowla and S. Chowla, Problems on periodic simple continued fractions, Proc. Nat. Acad. Sci. U.S.A. 69 (1972), 37-45.

2. G. H. Hardy and E. M. Wright, An introduction to the theory of numbers, 3rd ed., Clarendon Press, Oxford, 1954.

3. Oskar Perron, Die Lehre von den Kettenbruechen, Chelsea, New York, 1950.

Department of Mathematics, Brown University, Providence, Rhode Island 02912 